Recepción: 19 / 05 / 2018

Aceptación: 24 / 06 / 2018

Ciencias económicas y empresariales

Publicación: 15 / 09 / 2018

\title{
Gestión Administrativa Eficiente
}

\section{Efficient Administrative Management}

\section{Gestão Administrativa Eficiente}

\author{
Nelly G. Salguero-Barba ${ }^{\text {I }}$ \\ nelly.salguero@utc.edu.ec \\ Christian P. García-Salguero II \\ cristian.garciasalguero@mail.utoronto.ca
}

Correspondencia: nelly.salguero@utc.edu.ec

\footnotetext{
${ }^{\mathrm{I}}$ Magister en Planeamiento y Administración Educativos, Licenciada en Administración Ejecutiva, Docente de la Universidad Técnica de Cotopaxi, Latacunga, Ecuador.

II Universidad de Toronto, Canadá.
} 


\section{Resumen}

Propiciar una administración eficiente es estudiar la manera en que las empresas adquieren y utilizan sus recursos para cumplir objetivos y obtener beneficios. Debemos tomar en cuenta que para llevar a cabo un proceso administrativo eficiente, es necesario trazar metas, planificar estrategias y establecer políticas, de la mano de un proceso lógico y ordenado que permita cumplir planes, plazos y evidenciar resultados, cuyo propósito es disminuir el riesgo al fracaso, evitando errores y asegurando el éxito empresarial, integrando controles de gestión organizacionales, sin descuidar el rol fundamental que juega el recurso humano. Es necesario seleccionar indicadores que permitan monitorear, controlar y mejorar los ingresos del negocio, todo esto debe ir en función de alcanzar la satisfacción del cliente, que, por supuesto dependerá de la eficiencia del talento humano y el buen uso de los recursos técnicos, humanos, financieros. Lo ideal es innovar constantemente, tomando en cuenta que la competencia podría ganar mercado a través de los nuevos productos ofertados, adelántese siempre al futuro, venciendo miedos e incertidumbres. El propósito de este trabajo es identificar las herramientas necesarias para que la gestión administrativa se de en forma efectiva. Se concluye que para que una empresa funcione de manera eficiente, es imprescindible la planeación, organización, dirección y control, lo que contribuirá a lograr una sociedad económicamente estable.

Palabras claves: gestión administrativa; innovación; eficiencia del talento humano; éxito empresarial.

\section{Abstract}

To promote an efficient administration is to study the way in which companies acquire and use their resources to achieve objectives and obtain benefits. We must take into account that to carry out an efficient administrative process, it is necessary to set goals, plan strategies and establish policies, with a logical and orderly process that allows to meet plans, deadlines and evidence results, whose purpose is to reduce the risk to failure, avoiding mistakes and ensuring business success, integrating organizational management controls, without neglecting the fundamental role played by human resources. It is necessary to select indicators to monitor, control and improve business income, all this must be based on achieving customer satisfaction, which, of course, will depend on the efficiency of human talent and the proper use of technical, human resources, 
financial. The ideal is to innovate constantly, taking into account that the competition could gain market through the new products offered, always advance to the future, overcoming fears and uncertainties. The purpose of this work is to identify the necessary tools so that the administrative management is effectively carried out. It is concluded that for a company to function efficiently, planning, organization, direction and control are essential, which will contribute to achieving an economically stable society.

Keywords: administrative management; innovation; efficiency of human talent; Business success.

\section{Resumo}

Promover uma administração eficiente é estudar a maneira pela qual as empresas adquirem e usam seus recursos para alcançar objetivos e obter benefícios. Devemos levar em conta que, para realizar um processo administrativo eficiente, é necessário estabelecer metas, planejar estratégias e estabelecer políticas, com um processo lógico e ordenado que permita atender a planos, prazos e resultados de provas, cujo objetivo é reduzir o risco. Ao fracasso, evitando erros e garantindo o sucesso do negócio, integrando controles de gestão organizacional, sem negligenciar o papel fundamental desempenhado pelos recursos humanos. É necessário selecionar indicadores para monitorar, controlar e melhorar a renda dos negócios, tudo isso deve ser baseado na satisfação do cliente, o que, naturalmente, dependerá da eficiência do talento humano e do uso adequado dos recursos técnicos e humanos. Financeiro. O ideal é inovar constantemente, levando em conta que a concorrência poderia ganhar mercado através dos novos produtos oferecidos, sempre avançando para o futuro, superando medos e incertezas. O objetivo deste trabalho é identificar as ferramentas necessárias para que a gestão administrativa seja efetivamente realizada. Conclui-se que para que uma empresa funcione eficientemente, o planejamento, a organização, a direção e o controle são essenciais, o que contribuirá para o alcance de uma sociedade economicamente estável.

Palavras chave: gestão administrativa; inovação; eficiência do talento humano; sucesso nos negócios. 


\section{Introducción}

La gestión administrativa requiere de una adecuada toma de decisiones que permita a las empresas incursionar y mantenerse en un mercado competitivo. La presente investigación es bibliográfica, el método utilizado es el deductivo, a través del cual se sacaron las conclusiones y recomendaciones, se debe tomar en cuenta que un cambio en la gestión administrativa es posible siempre y cuando los administradores se empoderen de la empresa y manejen los procesos de manera eficiente y transparente.

El representante de la empresa debe ser un líder comprometido con los cambios vertiginosos que se nos ofrece y de la mano de la tecnología moderna, que le permita desarrollar un plan de trabajo efectivo con objetivos y metas realizables que promuevan una gestión oportuna y diligente, que garantice la solvencia y capacidad de crecimiento.

Hurtado (2008), manifiesta que la gestión administrativa es un proceso muy particular consistente en las actividades de planeación, organización, dirección, ejecución y control, desempeñadas para determinar y alcanzar los objetivos señalados con el uso de seres humanos y otros recursos.

Monchón. (2014), indica que la administración consiste en coordinar y supervisar las actividades de las organizaciones para lograr que éstas alcancen su objetivo de forma eficaz y eficiente. Las funciones básicas de la administración (planear, organizar, dirigir y controlar.) se dirigen alcanzar los propósitos establecidos por la organización. La labor de la administración deberá adaptarse a las características de cada organización, así como tener cuenta el entorno que influye en ella.

Hitt et al. (2006), definen a la administración como "el proceso de estructurar y utilizar conjuntos de recursos orientados hacia el logro de metas, para llevar a cabo las tareas en un entorno organizacional".

\section{Gestión Administrativa eficiente}

Cada Empresa es un mundo aparte y no en todas aplican las mismas estrategias a la hora de alcanzar el éxito. 


\section{Reglas de la Administración}

Planeación: diseñar un plan de acción para el mañana

Organización: brindar y movilizar recursos para la puesta en marcha del plan.

Dirección: dirigir, seleccionar y evaluar a los empleados con el propósito de lograr el mejor trabajo para alcanzar lo planificado.

Coordinación: integración de los esfuerzos y aseguramiento de que se comparta la información y se resuelvan los problemas.

Control: garantizar que las cosas ocurran de acuerdo con lo planificado y ejecución de las acciones correctivas necesarias de las desviaciones encontradas.

Terry George explica que la administración es un proceso distintivo que consiste en planear, organizar, ejecutar y controlar, desempeñada para determinar y lograr objetivos manifestados mediante el uso de seres humanos y de otros recursos.

Canovas Riveron "et-alt" (2011), manifiesta que la estructura que debe tener una organización para lograr la eficacia debe ser parte de un todo organizacional y de su estructura para garantizar la eficiencia de todas las partes involucradas, sean ellas órganos o personas. Fayol partió de un enfoque sintético, global y universal de la empresa, lo cual inició la concepción anatómica y estructural de la organización. Existe una proporcionalidad de la función administrativa, que se distribuye en todos los niveles de la empresa.

\section{Características de la organización funcional}

Para varios autores, la estructura sigue a la estrategia. Según A Chandler (1962),2 si una organización adopta una estrategia de expansión, debe dividir sus departamentos por funciones, en tanto si asume una estrategia de diversificación, la estructura debe ser multidivisional. Estudios posteriores realizados por diversos autores llegaron a la conclusión de que indudablemente existe una relación directa entre estructura y estrategia.3 En el modelo de Scot, por ejemplo, se establecen tres etapas de evolución de la empresa. El paso de una a otra etapa es 
consecuencia de un desarrollo hacia una diversificación.4 Las características de la organización cambian, y se modifica también la estructura organizacional, como resultado de las sucesivas estrategias adoptadas.

Según Menguzzato M y Renal JJ (1995), el paso de una estructura organizacional a otra no puede ser instantáneo; la estructura no es el único factor que influye en la estrategia; además, debe considerarse el liderazgo y la cultura de la organización.

Autoridad funcional o dividida. Es una autoridad que se sustenta en el conocimiento. Ningún superior tiene autoridad total sobre los subordinados, sino autoridad parcial y relativa.

Línea directa de comunicación. Directa y sin intermediarios, busca la mayor rapidez posible en las comunicaciones entre los diferentes niveles.

Descentralización de las decisiones. Las decisiones se delegan a los órganos o cargos especializados.

Énfasis en la especialización. Especialización de todos los órganos a cargo

\section{Empresa humanística}

El abandono de lo humano en la construcción de sociedad, empresa y Estado, se convierte en la causa natural de esta humanidad deshumanizada. Al respecto, cabe nombrar algunos aspectos que evidencian la crisis ética o debacle de humanidad en las organizaciones:

Los conflictos financieros y los escándalos corporativos de las grandes multinacionales, que arrastran conglomerados empresariales, negocios y con ellos a personas a la tragedia de la deshumanización.

El deseo de monopolio de muchas organizaciones que generan pobreza y frustran el ideario de desarrollo integral como alternativa a un modelo dominante de ganancias y superávit a costa de lo que fuere necesario. La paradoja del progreso reza: más oportunidades, más excluidos, menos garantías, menos equidad. 
Los incorrectos manejos y comportamientos deshonestos de los gobiernos corporativos, van dando origen a un escenario peligroso de corrupción donde el sentido profesional fácilmente se asocia al alcance del éxito a toda costa.

Los cuestionamientos a los sistemas financieros como "mano oscura" que tras bambalinas controlan los sistemas socio-políticos de los países y que limitan la capacidad per cápita de desarrollo y accesibilidad a las garantías sociales y económicas expuestas en los derechos humanos.

\section{Falta de comunicación e información}

\section{Gestión de la información}

La gestión de información ha permitido el uso de la información y el conocimiento en el diseño de servicios y productos para obtener ventajas competitivas. Cuando la información no se gestiona bien ocurren los siguientes problemas:

Se crea confusión en los funcionarios, debido al exceso de información innecesaria.

Se dificulta el acceso a la información dentro de la organización.

Existe una divulgación deficiente de la información, razón por la cual no circula.

Sobrecarga de información.

No se valora la información.

\section{Bajo nivel de uso y análisis de la información.}

\section{Inercia organizacional}

Divulgación y compartimentación de la información deficiente (Lage Machín L. Estudio para la propuesta de información a introducir y servicios a ofrecer en la intranet de CUPET. [Tesis para optar por el título de licenciado en Bibliotecología y Ciencias de la Información]. La Habana: Facultad de Comunicación, Universidad de La Habana; 1999. p.16.) 
Procesos lentos de toma de decisiones.

Duplicidad de la información.

\section{Conceptualización}

La administración como disciplina es relativamente nueva, la historia del pensamiento administrativo es muy antigua, ya que nace con el hombre mismo, puesto que en todo tiempo ha habido la necesidad de coordinar actividades, tomar decisiones y ejecutarlas; de ahí que en la administración antigua se encuentran muchos de los fundamentos administrativos de la actualidad y que pueden observarse en el código de Hammurabi, en el Nuevo Testamento, así como en la forma de conducir los asuntos en la antigua Grecia, Egipto, Roma y China, en donde se encuentran vestigios del proceso administrativo.

La evolución de la gestión administrativa se entiende en términos de cómo han resuelto las personas las cuestiones de sus relaciones en momentos concretos de la historia, prevaleciendo la gestión del talento humano.

Un trabajo eficiente y oportuno del talento humano aporta al adelanto empresarial, por tanto es necesario mantenerlos motivados y en constante capacitación y actualización de conocimientos, se debe propiciar una efectiva comunicación entre Directivos y empleados, lo que mejorará el desempeño laboral, delimitando cargos y responsabilidades, en función de los objetivos institucionales.

Precursores de la Gestión Administrativa

Confucio

Adam Smith

Henry Metacalfe

Woodrow Wilson

Federick W Taylor 
Henrry Fayol

\section{Principios de Fayol}

División del trabajo: cuando más se especialicen las personas, con mayor eficiencia desempeñaran su oficio.

Autoridad: los gerentes tienen que dar órdenes para que se hagan las cosas; si bien la autoridad formal les da derecho de mandar.

Disciplina: Los miembros de una organización tienen que respetar las reglas y convenios que gobiernan la empresa.

Unidad de dirección: Las operaciones que tienen un mismo objetivo deben de ser dirigidas por un mismo gerente que use un solo plan.

Unidad de mando: Cada empleado debe recibir instrucciones sobre una operación particular solamente de una persona.

Subordinación de interés individual al bien común: en cualquier empresa el interés de los empleados no debe tener prelación sobre los intereses de la organización como un todo.

Remuneración: la compensación por el trabajo debe ser equitativa para los patronos.

Centralización: Fayol creía que los gerentes deben conservar la responsabilidad final pero también necesitan dar a sus subalternos la autoridad suficiente para que puedan realizar adecuadamente su oficio.

Jerarquía: La línea e autoridad en una organización representada hoy generalmente por cuadros y líneas de un organigrama pasa en un orden de rango desde la alta gerencia hasta los niveles más bajos.

Orden: los materiales y las personas deben de estar en el lugar adecuado, en el momento adecuado, en particular, cada individuo debe ocupar el cargo o posición adecuados para él. 
Equidad: Los administradores deben ser amistosos y equitativos con sus subalternos.

Estabilidad del personal: una alta tasa de rotación del personal no es conveniente para el eficiente funcionamiento de una organización.

Iniciativa: debe darse a la subalterna libertad para concebir y llevar a cabo sus planes, aun cuando a veces se cometan errores.

Espíritu de Grupo: promover el espíritu de equipo dará a la organización un sentido de unidad.

\section{Metodología}

Se entiende por gestión al proceso emprendido por una o más personas para coordinar las actividades laborales de otras personas con la finalidad de lograr resultados de alta calidad que cualquier otra persona, trabajando sola, no podría alcanzar. En la gestión, los directivos utilizan ciertos principios que le sirven de guía en este proceso. También consideran la gestión como una disciplina, definiéndola como un cuerpo acumulado de conocimientos susceptibles de aprendizaje mediante el estudio.

Pacheco, Castañeda y Caicedo (2004), definen la gestión de toda institución como el hecho de realizar cosas por un grupo de hombres, con el objeto de obtener ciertos resultados de la manera más eficaz y económica posible. Consideran que la gestión es universal y aseguran la explotación óptima de los recursos. Igualmente, plantean que una gestión racional implica la identificación y previsión de las necesidades a satisfacer, la determinación de los objetivos, la búsqueda de las acciones más eficaces, la decisión de elegir las acciones a realizar considerando los recursos que se tengan en el presente y que puedan estar al alcance del futuro, ejecutar las decisiones y controlar la ejecución misma, fundamentándose en el logro de los objetivos y en la toma de decisiones. En efecto, la gestión es el proceso mediante el cual se formulan objetivos y lue go se miden los resultados obtenidos para finalmente orientar la acción hacia la mejora continua 


\section{Conclusiones}

Se concluye que para que una empresa funcione de manera eficiente, es imprescindible la aplicación de la planeación, organización, dirección y control, lo que contribuirá a lograr una sociedad efectiva y económicamente estable.

\section{Referencias Bibliográficas}

Amat, J. (2000). Control de Gestión (una perspectiva de dirección). Editorial Gestión. España.

Beltrán, J. (2003), Indicadores de gestión. 3R Editores. Bogotá. Colombia Blanco, I. (2000).

Berreiro Fernández, J., Diez de Castro, J., Barreiro Fernández, B., Ruzo Sanmartín, E., \& Lozada Pérez, F. (2003).

El Control Integrado de Gestión. Limusa Noriega Editores. México Goldratt, E. (2004).

Comba Paola (2013). Gestión Administrativa.

Gestión científica empresarial. Coruña: Netbiblo. Caldas, M., Reyes, C., \& Heras, A. (2009). Empresa e iniciativa emprendedora. Madrid: Editex. Campiña Domínguez, G., \& Fernández.

González López, L. (2007). Humanismo y gestión humana. Una perspectiva de interpretación para el trabajo social aplicado al campo laboral. Eleuthera, 42-63.

Guinart Josep María. (2008). Indicadores de gestión para las entidades públicas

Hernández, M. (2010). La empresa y su entorno. En G. Campiña Domínguez, \& M. J. Fernández

Hernández, Empresa y Administración (pág. 290). Madrid: Editex. Canovas.

Hernández Hugo (2011). La gestión empresarial, un enfoque del siglo XX, desde las teorías administrativas científica, funcional, burocrática y de relaciones humanas.

Indicadores Integrales de Gestión. Editorial Mc Graw Hill. Bogotá, Colombia. Chiavenato, I. (2005). Comportamiento Organizacional Editorial Thomson. México. 
La Meta. 20ava edición. Editorial castillo. México Méndez, E (1993). Gerencia Académica.

La construcción de la Educación del III. Milenio. Editorial de la Universidad del Zulia. Maracaibo Nava, H. (2004). La investigación jurídica ¿Cómo se elabora el proyecto? Maracaibo. Venezuela. 2da Ed. Ediluz Pacheco, J; Castañeda W y Caicedo Carlos Hernán (2004).

La gestión empresarial en el siglo XXI. Foro iberoamericano sobre estrategia de comunicación (págs. 1-28). Buenos Aires: FISEC. Contreras Soto, R. (2010).

Pérez Rodríguez Yudith2. (2007). Organización funcional, matricial... En busca de una estructura adecuada para la organización.

Riveron, G., Loredo Carballo, N., \& Martín Pérez, C. (2011). Evaluación del grado de aplicación de las teorías de dirección en empresas perfeccionadas del territorio. Observatorio de la Economía Latinoamericana. Castilla, A. (2005). Reflexiones en torno a la construcción científica en el campo de los estudios de las organizaciones, gestión y administración en México. Observatorio de la economía latinoamericana, 20-56. 